\title{
Quality of injectable oxytetracycline circulating in legal markets of Addis Ababa, Ethiopia using physio- chemical and sterility analysis
}

\author{
Yosef Nigussie ${ }^{1}$, Achenef Melaku ${ }^{1^{*}}$, Misgana Tadese ${ }^{2}$, Bizuayehu Belete ${ }^{2}$, and Elias \\ Kebede ${ }^{1}$ \\ ${ }^{1}$ Department of Veterinary Pharmacy, College of Veterinary Medicine and Animal Sciences, Uni- \\ versity of Gondar \\ ${ }^{2}$ Veterinary Drug and Feed Control and Administration Authority, Animal Products, Veterinary \\ Drug, and Feed Quality Assessment Centre, Addis Ababa \\ *Corresponding author: - Tel.: +251918065724; E-mail: achenefmela@yahoo.com
}

\begin{abstract}
This study was conducted to evaluate the quality of different brands of injectable oxytetracycline solutions circulating in the legal markets of Addis Ababa with respect to physicochemical characteristics and sterility. Nine brands of oxytetracycline with thirteen different batches were randomly purchased from veterinary drug stores in the city. The physical assessment was performed by using a checklist that was prepared based on the World Health Organization guidelines. The qualitative and quantitative analysis of active pharmaceutical ingredients (API) was performed by High-Performance Liquid Chromatography. The sterility test was assessed by using the direct inoculation method. All samples passed the identity, the assay, and the sterility tests. However, there were statistically significant differences $(P<0.05)$ among brands in the quantity of API. The highest percentage of the API was recorded in brand G $(112.12 \% \pm$ 1.86) while the lowest was seen in brand $\mathrm{H}(92.61 \% \pm 1.5)$. This study revealed that all brands passed both physicochemical and sterility tests except for the differences in the API level among brands. Strict regulation, monitoring, and wider-scale surveillance are required to assure sustainable control of substandard, unsterile, and falsified pharmaceutical products in the country.
\end{abstract}

Keywords: Batches; Brands; HPLC; Oxytetracycline; Physicochemical analysis; Sterility Test. 


\section{Introduction}

The quality of a drug is an integral part of a successful treatment regimen. To attain and sustain the required quality, there must be a system of quality assurance. Many developing countries do not have an effective means of monitoring the quality of generic drug products in the market. This results in the widespread distribution of substandard and counterfeit pharmaceutical products. Due to this, the World Health Organization (WHO) issued guidelines for global standards and requirements for the registration, assessment, marketing, authorization, and quality control of pharmaceutical products (Taylor et al., 2001; Glass, 2014; Kelesidis and Falagas, 2015; WHO, 2015).

Oxytetracycline (OTC), which exhibits broad-spectrum antimicrobial activity against a variety of microbes, is the most frequently used antimicrobial drug, particularly in veterinary medicine. Some formulations, however, have been reported ineffective against disease-causing organisms (Michaloa et al., 2004). This may be related to the quality of the drug or the development of resistance by the organisms. Reports showed that the counterfeit rate may reach up to $30 \%$ of drugs sold in Sub-Saharan Africa, of which antimicrobial agents have been the most popular ones (Glass, 2014; Kelesidis and Falagas, 2015; WHO, 2018). World Health Organization (2018) estimated that one in ten medical products in low and middle-income countries are substandard or falsified. Substandard products cause treatment failure, harm the patient, promote antimicrobial resistance, lead to loss of confidence, or trust in the healthcare system (Okeke et al., 2007; WHO, 2018).

A drug like oxytetracycline is mainly imported to Ethiopia by private companies in which manufacturers are located outside the country. Quality tests are usually conducted during importing. The borders of the country are porous which may allow the entrance of substandard drugs. Hence, periodic assessment of drugs circulating in the market will make the quality control process complete and help to design systems or strategies to control the circulation of substandard drugs. The authors of this article conducted a quality test on albendazole, a quite commonly used drug to treat helminthic parasites, and found that 3 of the 10 batches contained a lower amount of albendazole per tablet than expected (Seifu et al., 2019). This finding initiates us to test the quality of other drugs like oxytetracycline which is quite commonly sold and used drug for the treatment of widespread infectious diseases in animals (Tufa et al., 2018). To the best of our knowledge, there was no information in the 
country concerning the quality and sterility of oxytetracycline (OTC) circulating in the market. Therefore, this study was conducted to evaluate the physicochemical quality and sterility of injectable OTC solutions circulating in the legal market of Addis Ababa, Ethiopia.

\section{Materials and methods}

\section{Study area}

The study was conducted in Addis Ababa which is the capital city and administrative centre for the Federal Democratic Republic of Ethiopia. The city lies at $9.03^{\circ}$ North latitude and $38.8^{\circ}$ East longitudes (UN-Habitat, 2017). The city is the hub for the production, importation, and distribution of veterinary pharmaceuticals.

\section{Study design and sampling}

A cross-sectional study was conducted from November 2018 to April 2019. Thirteen brands of oxytetracycline injectable solution were included in the study and two vials were used for each brand for assessment. Drugs were purchased from randomly selected veterinary pharmacies in the city. During sampling, safety protocols were considered to avoid factors that reduce the quality of the drugs which means that purchased drugs samples were transported and stored as per the recommendation of the manufacturers. All laboratory activities were performed in Animal Products, Veterinary Drug, and Feed Quality Assessment Centre, which is located in Akaki Kality sub-city, Addis Ababa, Ethiopia.

\section{Physicochemical analysis}

High-Performance Liquid Chromatography (HPLC) (Agilent Technologies, Japan) was used for the identification and quantification of API in the samples. HPLC graded chemicals and reagents like methanol, orthophosphoric acid, acetonitrile, hydrochloric acid, oxytetracycline reference standard (USP, USA), triethylamine, and ultrapure deionized water were used for the analysis.

The mobile phase was prepared by mixing $4.075 \mathrm{ml}$ of orthophosphoric acid in $1000 \mathrm{ml}$ of ultrapure deionized distilled water and the $\mathrm{pH}$ of the solution was adjusted to 2.7 with triethylamine. Then, $650 \mathrm{ml}$ of this solution was mixed 
with $100 \mathrm{ml}$ of methanol and $250 \mathrm{ml}$ of acetonitrile, sonicated for 30 minutes, and passed through a filter with $0.5 \mu \mathrm{m}$ pore size.

Oxytetracycline (OTC) $(1 \mathrm{mg} / \mathrm{ml})$ reference standard (USP) was prepared by taking two OTC reference standards (ref1 and ref2). For both, $0.025 \mathrm{~g}$ was accurately weighed and transferred to a $25 \mathrm{ml}$ volumetric flask and dissolved in a diluting solution, and mixed by vortexing. Then, $10 \mathrm{ml}$ of this stock solution was transferred into another $100 \mathrm{ml}$ volumetric flask, and further diluted with distilled water to the required volume, and then transferred to HPLC vials after the syringe filter.

During the preparation of the sample, two vials of OTC of the same batch and brand were added into one beaker and mixed well. Then, an equivalent of 1 $\mathrm{mg} / \mathrm{ml}$ concentration of reference standards was calculated depending on the percent concentration of API of the sample (10 and $20 \%$ ), and then the sample was diluted to half and sonicated for a few minutes. Then, $10 \mathrm{ml}$ from the stock solution was taken and diluted to the volume by deionized distilled water and mixed well again. Afterward, the assay preparation was taken into a syringe, and passed through a $0.45 \mu \mathrm{m}$ syringe filter, and filtered into $75 \%$ of $1.5 \mathrm{ml}$ HPLC vial, and it was ready for determination assay.

The HPLC system was equipped with a UV-vis detector (SPD-20A/20AV, Shimadzu Corporation, Japan), a $254 \mathrm{~nm}$ detection wavelength, and a $4.6 \mathrm{~mm}$ by $25 \mathrm{~cm}$ column that contains $5 \mu$ particle size. The flow rate was about one $\mathrm{ml}$ per minute. The total chromatography run time was 12 minutes. Before running the samples, the suitability, and stability of the system were tested (USP, 2012).

An equal volume (about $10 \mu \mathrm{l}$ ) of the standard and the sample preparations were injected into the chromatograph separately. The chromatograph was recorded and measured for the response of peaks. For the identification, we compared the retention time of the OTC sample with that of the standard (Kupiec, 2004). The peak areas of the standard and the sample were used for quantification (Van Tonder et al., 1996). All procedures were conducted three times to increase the precision.

According to the official compendia of USP, OTC for injection contains an amount of OTC Hydrochloride equivalent to not less than $90 \%$ and not more than $120 \%$ of the labelled amount of oxytetracycline (USP, 2015). 


\section{Sterility testing}

Thioglycollate and soybean casein digest media were used for sterility testing. It was carried out by following basic microbiological aseptic techniques in the biosafety label II laboratory. The thioglycollate was used to test the growth of bacteria. Soya-bean casein digest was performed to test whether fungi or aerobic bacteria grew or not. Fluid thioglycolate was incubated at $35^{\circ} \mathrm{C}$ whereas the Soybean casein digest was incubated at $25^{\circ} \mathrm{C}$.

The inoculation was conducted after making a serial dilution of Oxytetracycline up to $10^{-6}$ using a soybean digest medium. From each diluted tube, one $\mathrm{ml}$ of the fluid was transferred to the prepared media. Two-stage incubation was conducted to increase the accuracy and visibility. First, the sample was inoculated in the media and incubated for 14 days. After that one $\mathrm{ml}$ of the mixed fluid was taken and inoculated into the new media of the same type and incubated for an additional 4 days. In all cases, media without a drug was incubated and used as the negative control. As a positive control, thioglycolate was incubated with drug and bacteria (Staphylococcus aureus), and soybean casein digest was inoculated with diluted drug and fungi (Candida albicans). The interpretation was made based on the absence or presence of turbidity (Amrutha et al., 2017).

\section{Ethical consideration}

An agreement was reached between the investigators and local veterinary pharmacists, after explaining the objective of the research. The owners agreed to sell the drugs with a full description as they are coming from the manufacturer or distributor. During sampling, the product manufacturer, brand name, and name of the pharmacy or owner were recorded and coded for anonymity. Hence, the brands were coded in the preparation of this manuscript.

\section{Data analysis}

The data were checked, coded, and entered into Microsoft Excel 2010. Descriptive statistics like percentage mean and relative standard deviation (RSD) were used to express and summarize the data. The content of API in each brand was compared by using a one-way analysis of variance (ANOVA). The Least Square Difference (LSD) was used to conduct pairwise comparisons. A $P$-value of less than 0.05 was considered statistically significant. 


\section{Results}

\section{Packaging information}

All brands and batches fulfilled the criteria in terms of container character, strength (concentration), dosage statement, storage information, batch number, or lot number, manufactory, and expiry date. However, 2 (22.2\%) brands and batches $3(23.1 \%)$ had no leaflet or package insert (Table 1$)$.

Table 1. Physical assessment of oxytetracycline

\begin{tabular}{|c|c|c|c|c|c|c|c|}
\hline Product & $\begin{array}{l}\text { Container } \\
\text { and closer }\end{array}$ & $\begin{array}{l}\text { API con } \\
\text { centration } \\
(\mathrm{mg} / \mathrm{mL})\end{array}$ & $\begin{array}{l}\text { Dosage } \\
\text { statem } \\
\text { ent }\end{array}$ & $\begin{array}{l}\text { Storage } \\
\text { informa } \\
\text { tion }\end{array}$ & $\begin{array}{l}\text { Leaflet } \\
\text { or } \\
\text { package } \\
\text { insert }\end{array}$ & $\begin{array}{l}\text { Batch } \\
\text { Number }\end{array}$ & $\begin{array}{l}\text { Manufa } \\
\text { ctured } \\
\text { and Exp } \\
\text { ired Date }\end{array}$ \\
\hline Brand A & Yes & Yes & Yes & Yes & Yes & Yes & Yes \\
\hline Brand B1 & Yes & Yes & Yes & Yes & Yes & Yes & Yes \\
\hline Brand B2 & Yes & Yes & Yes & Yes & Yes & Yes & Yes \\
\hline Brand C & Yes & Yes & Yes & Yes & Yes & Yes & Yes \\
\hline Brand D1 & Yes & Yes & Yes & Yes & Yes & Yes & Yes \\
\hline Brand D2 & Yes & Yes & Yes & Yes & Yes & Yes & Yes \\
\hline Brand E1 & Yes & Yes & Yes & Yes & $\mathrm{No}^{*}$ & Yes & Yes \\
\hline Brand E2 & Yes & Yes & Yes & Yes & $\mathrm{No}^{*}$ & Yes & Yes \\
\hline Brand F1 & Yes & Yes & Yes & Yes & Yes & Yes & Yes \\
\hline Brand F2 & Yes & Yes & Yes & Yes & Yes & Yes & Yes \\
\hline Brand G & Yes & Yes & Yes & Yes & Yes & Yes & Yes \\
\hline Brand $\mathrm{H}$ & Yes & Yes & Yes & Yes & $\mathrm{No}^{*}$ & Yes & Yes \\
\hline Brand I & Yes & Yes & Yes & Yes & Yes & Yes & Yes \\
\hline
\end{tabular}

\section{Identification test}

An identity test was conducted by comparing the retention time of the sample with that of the standard in the chromatographic response (Table 2 and Figure $1)$. 
Table 2. Comparison of chromatographic retention time values of different brands of Oxytetracycline with a reference standard

\begin{tabular}{lcccl}
\hline Product & $\begin{array}{l}\text { Concentra } \\
\text { tion(mg/ } \\
\text { ml) }\end{array}$ & $\begin{array}{l}\text { Retention } \\
\text { time, minutes } \\
\text { (sample), }\end{array}$ & $\begin{array}{l}\text { Retention time } \\
\text { minutes (standard) }\end{array}$ & Identity test \\
\hline Brand A & 200 & 3.33 & 3.29 & Pass \\
Brand B1 & 100 & 3.27 & 3.29 & Pass \\
Brand B2 & 200 & 3.26 & 3.29 & Pass \\
Brand C & 200 & 3.33 & 3.29 & Pass \\
Brand D1 & 100 & 3.28 & 3.29 & Pass \\
Brand D2 & 200 & 3.29 & 3.29 & Pass \\
Brand E1 & 100 & 3.20 & 3.29 & Pass \\
Brand E2 & 200 & 3.20 & 3.29 & Pass \\
Brand F1 & 100 & 3.27 & 3.29 & Pass \\
Brand F2 & 200 & 3.26 & 3.29 & Pass \\
Brand G & 200 & 3.29 & 3.29 & Pass \\
Brand H & 100 & 3.33 & 3.29 & Pass \\
Brand I & 100 & 3.29 & 3.29 & Pass \\
\hline
\end{tabular}
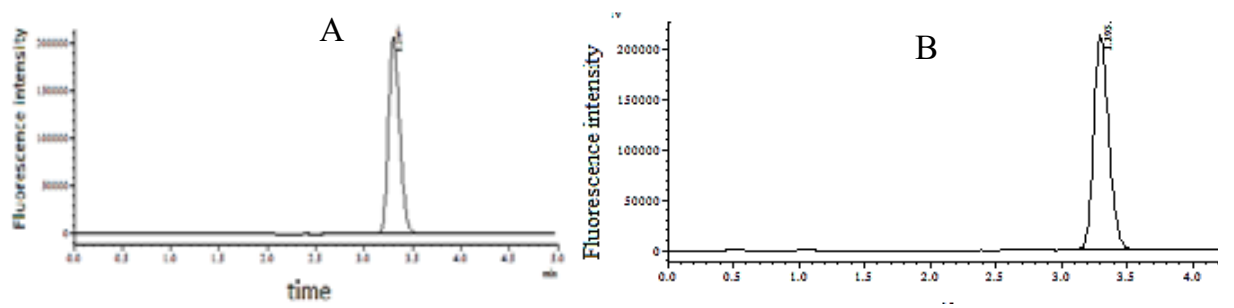

Figure 1. Representative image of the chromatograph of the standard (A) and one of the samples (B)

\section{Assay of oxytetracycline}

The results for the mean percentage label claim of the different brands of parenteral oxytetracycline preparation included in the study are depicted in Table 3. 
Nigussie et al.,

Table 3. The assay of tested oxytetracycline brands

\begin{tabular}{lcccl}
\hline Product & $\begin{array}{l}\text { Labelled } \\
\text { concentration } \\
\text { (mg/ml) }\end{array}$ & $\begin{array}{l}\text { Assay (mean \% } \\
\text { RSD) }\end{array}$ & $\begin{array}{l}\text { Content (mg) } \\
\text { obtained }\end{array}$ & $\begin{array}{l}\text { Assay test } \\
\text { decision }\end{array}$ \\
\hline Brand A & 200 & $102.15 \pm 0.03$ & 204.3 & Pass \\
Brand B1 & 100 & $96.71 \pm 1.03$ & 96.71 & Pass \\
Brand B2 & 200 & $98.82 \pm 0.23$ & 197.64 & Pass \\
Brand C & 200 & $97.64 \pm 1.49$ & 195.28 & Pass \\
Brand D1 & 100 & $106.34 \pm 1.12$ & 106.34 & Pass \\
Brand D2 & 200 & $102.60 \pm 1.45$ & 205.2 & Pass \\
Brand E1 & 100 & $100.62 \pm 1.14$ & 100.62 & Pass \\
Brand E2 & 200 & $98.41 \pm 1.57$ & 196.82 & Pass \\
Brand F1 & 100 & $100.88 \pm 0.13$ & 100.88 & Pass \\
Brand F2 & 200 & $103.75 \pm 0.79$ & 207.5 & Pass \\
Brand G & 200 & $112.12 \pm 1.86 *$ & 224.24 & Pass \\
Brand H & 100 & $92.61 \pm 1.50 *$ & 92.61 & Pass \\
Brand I & 100 & $96.45 \pm 0.79$ & 96.45 & Pass \\
*Acceptable range is between $90 \%$ and $120 \% *$ RSD & & relative standard deviation &
\end{tabular}

*Acceptable range is between $90 \%$ and $120 \%,{ }^{*} \mathrm{RSD}=$ relative standard deviation

Even though all brands and batches fulfilled both qualitative and quantitative criteria, there was a significant difference $(P<0.05)$ among the brands. The highest mean content $(112.12 \pm 1.86)$ and least mean $(92.61 \pm 1.50)$ contents were seen in brands $\mathrm{G}$ and $\mathrm{H}$, respectively (Table 3 ).

\section{Sterility testing}

All the brands passed the sterility testing which showed that they were free from aerobic bacteria and fungi (Table 4). 
Table 4. The sterility test result of oxytetracycline

\begin{tabular}{llll}
\hline Product & $\begin{array}{l}\text { Concentration } \\
(\mathbf{m g} / \mathbf{m l})\end{array}$ & $\begin{array}{l}\text { Soya bean } \\
\text { casein digest } \\
\text { medium }\end{array}$ & Thioglycolate medium \\
\hline Brand A & 200 & NT & NT \\
Brand B1 & 100 & NT & NT \\
Brand B2 & 200 & NT & NT \\
Brand C & 200 & NT & NT \\
Brand D1 & 100 & NT & NT \\
Brand D2 & 200 & NT & NT \\
Brand E1 & 100 & NT & NT \\
Brand E2 & 200 & NT & NT \\
Brand F1 & 100 & NT & NT \\
Brand F2 & 200 & NT & NT \\
Brand G & 200 & NT & NT \\
Brand H & 100 & NT & NT \\
Brand I & 100 & NT & NT \\
*NT= No turbidity (no bacterial or fungal growth) & \\
\hline
\end{tabular}

\section{Discussion}

The use of drugs in food-producing animals is fundamental for their health and productivity. To be effective a drug should have the required physicochemical qualities and should be sterile. The quality of a drug might be affected by the storage conditions, humidity, packaging materials, transportation, formulation, the nature of the active ingredient, and the presence of microorganisms (Othman, 2017; WHO, 2019). The pharmaceutical products should constitute not only an adequate amount of API but also must be packed with proper packaging materials in the desired container, which protects environmental conditions that may affect the quality of the products. Besides, the drug should be labelled well and have the leaflet inserted for additional information. In this study, $22.2 \%$ of the brands or $23.1 \%$ of batches didn't have leaflets or package inserts. The absence of leaflet was also reported by Seifu et al. (2019) for albendazole in the same study area. This may lead to the inappropriate use of the drugs. Misuse or inappropriate use of drugs may have a direct impact on the recipient and an indirect effect on future treatment practices through the development of drug resistance (Clifford et al., 2018). 
In this study, all brands of oxytetracycline passed the quality assessment and the identification test. These indicated that oxytetracycline in the market in the study area had good quality during the study period which may be due to good manufacturing practices followed by the storage and transportation of the products in the proper conditions. The periodical assessment and effective control at the point of entry for imported drugs are needed to keep the quality and avoid the circulation of substandard or falsified drugs in the market. The result obtained in this study was also in line with the study conducted in Ghana (Owusuwaa, 2016), that reported all seven injectable oxytetracycline investigated brands passed the assay quantification test. Contrary to our finding, poor quality of tetracycline capsules was reported in Nigeria (Okeke and Lamikanra, 1995); the difference may be related to the control system and the practices in manufacturing processes and drug handling conditions.

In this study, the statistical analysis of the mean differences in drug content revealed that the mean API content of different brands was significant $(P<0.05)$. The highest percentage content was obtained from brand G $(112.12 \% \pm 1.86)$ while the lowest drug content was obtained from brand $\mathrm{H}(92.61 \% \pm 1.50)$. The reason for the relative difference in the mean OTC assay between the brands might have been related to the pharmaceutical manufacturer's formulation condition. Variations in the composition of ingredients may be related to the amount of excipients and API added to the products (Amrutha et al., 2017). If the drug has higher API content than expected, the side effects may be intensified whereas if it contains a lower amount than expected, it will not be effective and may favor the development of drug resistance.

All forms of therapeutic products are expected to be sterile that is free of viable microorganisms. Principally, these include parenteral, ophthalmic, and irrigational preparations. Of these, parenteral products are unique dosage forms of drugs as they are injected subcutaneously, intramuscularly, or intravenously into the internal body compartments. As they have circumvented the highly efficient first line of body defense like the skin or mucous membranes, they must be free from microbial contamination (Amrutha et al., 2017).

The sterility tests are intended for detecting the presence of viable microorganisms in a pharmaceutical preparation that is designed to be sterile (USP, 2012). The test was based on the principle that if microbial contaminants are available, they can grow in a suitable media and their presence will be indicated by the formation of turbidity in the medium. To produce therapeutic effects 
without predisposing the patients to other infections, the parental drug should be sterile. This is due to the possibility of the presence of drug-resistant bacteria that can survive in drugs and grow when conditions become suitable. The contamination may occur during the manufacturing process from the equipment, person, or dust (Arzai, 2008). All brands of oxytetracycline included in this study were free from aerobic bacteria and fungi. Contrary to our current finding, Arzai (2008) reported bacteria and fungi contamination of OTC ointments in Nigeria.

\section{Conclusions}

In summary, the physicochemical evaluation showed that all of the brands tested complied with the pharmacopeia specifications described in the USP. However, the statistical comparison for drug content indicated a significant difference among brands. Besides, sterility testing also showed the absence of both bacterial and fungal contaminants. Despite these favorable findings, strict regulations, monitoring, and surveillance are required to assure sustainable safety, quality, and efficacy of drugs in the country.

\section{Abbreviations}

API: Active Pharmaceutical Ingredient; HPLC: High-Performance Liquid Chromatography; OTC: Oxytetracycline, RSD: Relative Standard Deviation; SD: Standard Deviation; USP: United States Pharmacopeia; UVS: UltravioletVisible Spectroscopy; WHO: World Health Organization

\section{Acknowledgments}

The authors would like to thank the technical staff working in National Animal Products, Veterinary Drugs, and Feed Quality Assessment Centre for their technical supports. We would also like to thank the authorities in the laboratory for allowing us to use the facilities in the laboratory for the investigation. 


\section{References}

Amrutha, A., Gangadharappa, H. V., Kiran, H. C., Shuchithra, S., 2017. In-process and finished products quality control tests for sterile and nonsterile dosage forms. Int. J. Pharm. Sci. Rev. Res. 45, 206-214.

Arzai, A. H., 2008. Evaluation of microbiological purity of some brands of tetracycline sold in Kano state, Nigeria. Bay. J. Pur. Appl.Sci., 1, 104-107.

Clifford, K. Desai, D., da Costa, C. P., Meyer, H., Klohe, K., Winkler, A. S., et al.., 2018. Antimicrobial resistance in livestock and poor quality veterinary medicines. Bull. Wrld. Hlth. Organ., 96, 662-664.

Glass, B., 2014. Counterfeit drugs and medical devices in developing countries. Res. Rep. Trop. Med., 5, 11.

Kelesidis, T. and Falagas, E., 2015. Substandard/counterfeit antimicrobial drugs. Clin. Microbiol. Rev., 28, 443-464.

Kupiec, T., 2004. Quality-control analytical methods: high-performance liquid chromatography. Int. J. Pharm. Comp., 8, 223-227.

Michaloa, E., Novotna, P. and Schlegelova, J., 2004. Tetracyclines in veterinary medicine and bacterial resistance to them. Vet. Med. Czech. 2004, 79-100.

Okeke, I. and Lamikanra, A., 1995. Quality and bioavailability of tetracycline capsules in a Nigerian semi-urban community. Int. J. Antimicrob. Agents., 5, 245-250.

Okeke, I. N. Aboderin, O. A., Byarugaba, D. K., Ojo,K. K., and Opintan, J. A., 2007. Growing problem of multidrug-resistant enteric pathogens in Africa. Emerg. Infect. Dis. 13, 1640-1646.

Othman, G., 2017. Fighting fake medicines: Quality evaluation of seven brands of albendazole tablets marketed in Yemen. Yemeni. J. Med. Sci., 11, 46-52.

Owusuwaa, A. A., 2016. Quality assessment of veterinary pharmaceuticals: A case of oxytetracycline and praziquantel. MSc Thesis, Kwame Nkrumah University of Science and Technology, College of Health Science Kumasi, Ghana.

Seifu, A., Kebede, E., Bacha, B., Melaku, A. and Setegn T., 2019. Quality of albendazole tablets legally circulating in the pharmaceutical market of Addis Ababa, Ethiopia: physicochemical evaluation. BMC Pharmacol. Toxicol. 5, 1-7.

Taylor, R.B.,Shakoor O., Behrens R. H., Everard, M., Low A.S., Wangboonskul, J., Reid, R G., Kolawole, J.A., 2001. Pharmacopoeial quality of drugs supplied by Nigerian pharmacies. The Lancet, 357, 1933-1936. 
Tufa, T. B., Gurmu, F., Beyi, A. F., Hogeveen, H., Beyene T. J., Ayana, D., et al., 2018. Veterinary medicinal product usage among food animal producers and its health implications in central Ethiopia. BMC Vet. Res., 14, 409.

UN-Habitat, 2017. The State of Addis Ababa 2017, the Addis Ababa we want, http:// mirror.unhabitat.org/downloads/docs/9173_97089_Addis_AbabFinal.pdf.

USP, 2012. Oxytetracycline Hydrochloride, Official Monograph, https://www.drugfuture.com/Pharmacopoeia/usp35/PDF/4188-4189\%20Oxytetracycline\%20Hydrochloride.pdf', pp. 4188-4189.

USP, 2015. United state pharmacopeia (USP). National formulary USP official Monograph, USP 38 NF 33 1, Pp.221-290. The United States pharmacopoeial convention 12601 Twin Book Parkway, Rockville, MD 20852.

VanTonder, E. C., de Villiers, M. M., Handford, J. S. and Malan, C. E., 1996. Simple, robust, and accurate high-performance liquid chromatography method for the analysis of several anthelmintics in veterinary formulations, J. Chromatogr. A. $729,267-272$.

WHO, 2015. Regional strategy for improving access to essential medicines in the Western Pacific Region, 2005 -2010. Available at: https://apps.who.int/iris/handle /10665/138155

WHO, 2018. Substandard and falsified medical products, Available at: https://www. who.int/news-room/fact-sheets/detail/substandard-and-falsified-medical-products.

WHO, 2019. World Health Organization, Essential Medicines and Health Products Information Portal, Available at: https://apps.who.int/medicinedocs/en/d/ Js2206e/7.3.html. 